\title{
Moment Method \& Pixel-by-Pixel Method: Complementary Mode Identification I. Testing FG Vir-like pulsation modes
}

\author{
W. Zima ${ }^{1}$, K. Kolenberg ${ }^{1,2}$, M. Briquet ${ }^{2}$, and M. Breger ${ }^{1}$ \\ 1 Institut für Astronomie, Türkenschanzstraße 17, 1180 Vienna, Austria \\ ${ }^{2}$ Instituut voor Sterrenkunde, Celestijnenlaan 200B, 3001 Heverlee, Belgium
}

\begin{abstract}
We have carried out a Hare-and-Hound test to determine the reliability of the Moment Method (Briquet \& Aerts 2003) and the Pixel-by-Pixel Method (Mantegazza 2000) for the identification of pulsation modes in Delta Scuti stars. For this purpose we calculated synthetic line profiles, exhibiting six pulsation modes of low degree and with input parameters initially unknown to us. The aim was to test and increase the quality of the mode identification by applying both methods independently and by using a combined technique. Our results show that, whereas the azimuthal order $m$ and its sign can be fixed by both methods, the degree $\ell$ is not determined unambiguously. Both identification methods show a better reliability if multiple modes are fitted simultaneously. In particular, the inclination angle is better determined. We have to emphasize that the outcome of this test is only meaningful for stars having pulsational velocities below $0.2 v \sin i$. This is the first part of a series of articles, in which we will test these spectroscopic identification methods.
\end{abstract}

\section{Introduction}

High-resolution spectroscopy is a powerful tool to identify the harmonic degree $\ell$ and the azimuthal order $m$ of a non-radially oscillating star by analyzing its line profile variations. In addition, the developed techniques enable the determination of the aspect angle of the pulsation/rotation axis, the pulsational temperature variations, and the intrinsic oscillation amplitude. In the past five years, considerable effort has been undertaken to develop and test reliable mode 
identification methods for spectroscopy, leading to a multitude of different techniques. The most promising spectroscopic approaches towards reliable mode diagnoses were acquired by the Moment Method (Aerts 1996, Briquet \& Aerts 2003), by studying amplitude and phase across the line (Schrijvers \& Telting 1999), and by direct line profile fitting (Mantegazza 2000). For low-degree modes of stars with narrow lines such as FG Vir and 44 Tau, these methods can yield a precise value for the azimuthal order $m$ and constrain the degree $\ell$.

\section{The Modeled Spectra}

The line profiles we used for this analysis were computed by integrating over the pulsational velocity field on the stellar surface, consisting of a 64800-element grid and taking a Gaussian as intrinsic line profile. The resulting velocities were calculated by summing up the contribution of every single pulsation mode. The model is based on the descriptions given by Schrijvers \& Telting (1997) and Balona (2000) and takes into account the effect of the Coriolis force, first and second order limb darkening, and temperature variations. All stellar parameters as well as the frequency domain of the modeled modes resemble those of the Delta Scuti star FG Vir (Breger 1998, 1999).

\begin{tabular}{|c|c|c|c|c|}
\hline & & & & \\
\hline$M$ & $1.85 M_{\odot}$ & & & \\
\hline$a$ & 0.43 (1st or & der I & $\mathrm{nb}$ & arkening) \\
\hline$b$ & 0.24 (2nd o & der & $\mathrm{mb}$ & larkening) \\
\hline Inclination $i$ & $=16^{\circ}$ & & & \\
\hline$v \sin i$ & $=22.8 \mathrm{kms}^{-1}$ & & & \\
\hline EQW & $=6.45 \mathrm{kms}^{-1}$ & (equ & ivale & tt width) \\
\hline$\sigma$ & $=7.67 \mathrm{kms}^{-1}$ & (wid & th of & intrinsic profile) \\
\hline name & Frequency $d^{-1}$ & $\ell$ & $m$ & $\frac{d R}{R}$ \\
\hline$\overline{f_{1}}$ & 12.716 & 1 & 0 & 0.00150 \\
\hline$f_{2}$ & 12.792 & 1 & 0 & 0.00050 \\
\hline$f_{3}$ & 20.288 & 2 & 1 & 0.00040 \\
\hline$f_{4}$ & 21.052 & 3 & -1 & 0.00044 \\
\hline$f_{5}$ & 9.200 & 1 & 1 & 0.00081 \\
\hline$f_{6}$ & 19.226 & 3 & -2 & 0.00040 \\
\hline
\end{tabular}

Table 1: Physical parameters of the input model for the test described in this paper.

To ensure an unbiased analysis, the six pulsation modes were given random values of a low degree $\ell$, azimuthal order $m$, and velocity amplitude. Addition- 
ally, the inclination $i$, the projected rotational velocity $v \sin i$, and the equivalent width EQW were set randomly. All values were saved in a file and compared to the outcome of the analyses. In order to create conditions as realistic as possible, we generated 561 line profiles, taking the same times of observation as of the 2002 FG Vir campaign of the Delta Scuti Network (Zima et al. 2003). Moreover, to reproduce the effect of phase smearing, we simulated an integration time of 7 minutes, which is $6 \%$ of the period of the main mode at $12.716 \mathrm{~d}^{-1}$. The noise of these observational data, consisting of the residuals of a cross correlation function after prewhitening with 15 frequencies, was added to the synthetic profiles (see Fig. 1).

\section{Mode Identification with the Pixel-by-Pixel Method}

The first mode identification technique we tested makes use of the IPS-Method developed by Telting \& Schrijvers $(1997,1999)$ and the Pixel-by-Pixel Method introduced by Mantegazza (2000). Briefly summarized, the pulsational content of every single oscillation mode is extracted, treated as mono-mode pulsation and fitted with theoretical profiles by means of a genetic algorithm.

The search for pulsation frequencies was performed by a package of computer programs, making use of Fourier techniques as well as of multiple frequency leastsquares algorithms, which fit a number of sinusoidals simultaneously to the data and do not rely on prewhitening. We examined the variations of the radial velocities (first moment) as well as of the pixel-by-pixel variations across the line. The latter is especially important to be examined, because it is possible that higher order modes can only be detected in the Fourier analysis of the pixel-by-pixel variations. As can be seen in Fig. 2, the modes $f_{4}$ and $f_{6}$ cannot be detected from the Fourier analysis of the radial velocities (right panel), whereas these modes reach a signal-to-noise ratio (SNR) of almost 4 in the pixel-to-pixel variations (left panel). We assumed that all

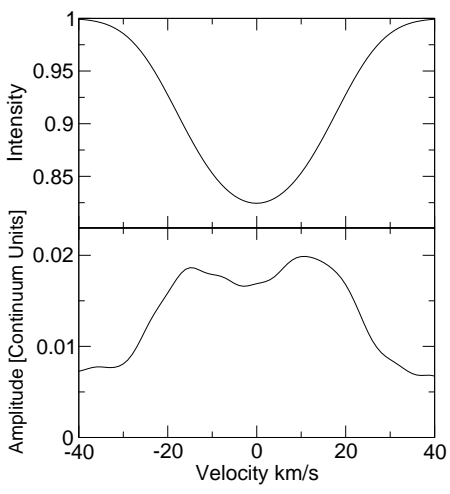

Figure 1: Average line profile (upper panel) and deviation from the mean of every single pixel (lower panel). The deviation of the pixels of 0.007 continuum units at the rim of the line implies an average signal-to-noise of about 140 . six modes are known with high precision from photometry, as is also the case for FG Vir, and thus relaxed the signal to noise criterion, which sets the limit for the significance of a peak in the Fourier spectrum to $S N R>4$ (Breger 1993).

In the next step, for every single detected pulsation frequency, the amplitude- 
and phase distribution across the line was calculated by deriving these values from a multi-periodic least squares fit for every velocity bin. The resulting diagrams (Fig. 3) already allow an estimate of the sign and value of $m$ from the left to right phase change, and thus decrease the number of free parameters for the subsequent fitting procedure. Evidently, $f_{1}$ and $f_{2}$ have axisymmetric character, which can be derived from the zero amplitude at the center of the line and a corresponding phase shift of $\pi$. Moreover, $f_{3}$ has an azimuthal order larger than 0 , due to its positive red-to-blue phase shift. We used this information to limit the number of free parameters and thereby speeding up the fitting procedure.

Hereafter, the decomposed line profiles were transformed back from Fourier space to real space, and 10 mono-mode profiles, evenly distributed across one pulsation cycle, were calculated for every single mode. The motivation for not directly fitting the parameters in Fourier space is mainly a time-saving one, because it is necessary to calculate much more line profiles in order to convert them properly to the Fourier domain.

The optimization was performed by means of a genetic algorithm, which minimizes the deviation parameter $\Gamma$ calculated from the sum of the squared difference between the ten "observed" spectra and ten theoretically computed
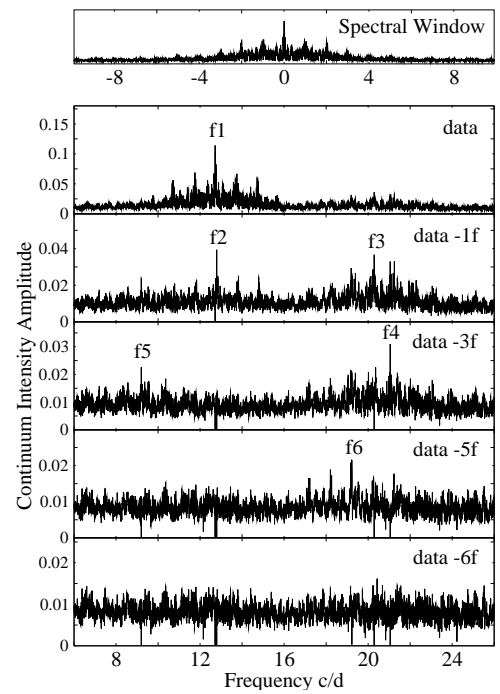
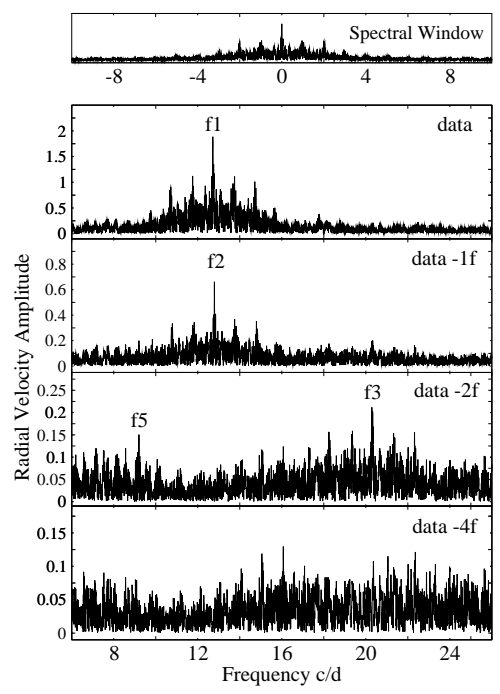

Figure 2: Fourier spectra of the pixel-by-pixel variations summed across the line (left panel) and the radial velocity (right panel). Every panel shows the data prewhitened of all frequencies which are marked above. 

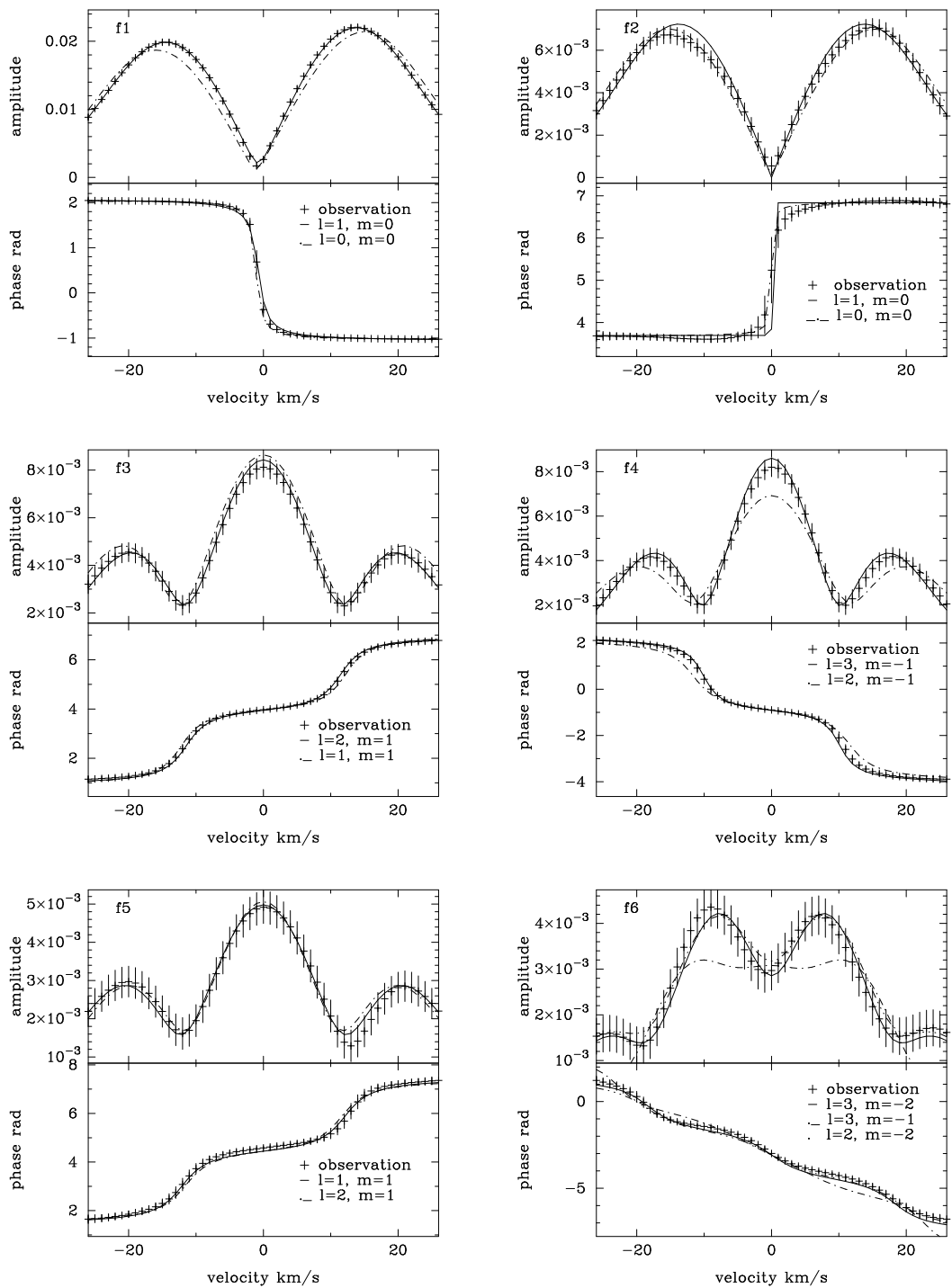

Figure 3: Amplitudes in continuum units and phases of the "observations" and the two best fits, which were derived by the Pixel-by-Pixel Method (Table 2) across the line for all six pulsation modes. For most modes the azimuthal number $m$ is much better constrained than the degree $\ell$, which is mainly due to the low inclination angle. 
spectra. Usually, the calculation of more than 10000 models and a number of iterative fitting procedures were needed to achieve a meaningful result for each mode. The advantage of a genetic algorithm in contrast to a grid is its good coverage of a large parameter space in much shorter calculation time. A persistent problem is that a confidence interval of the Chi-Square-test can not be defined, causing ambiguities in the mode identification.

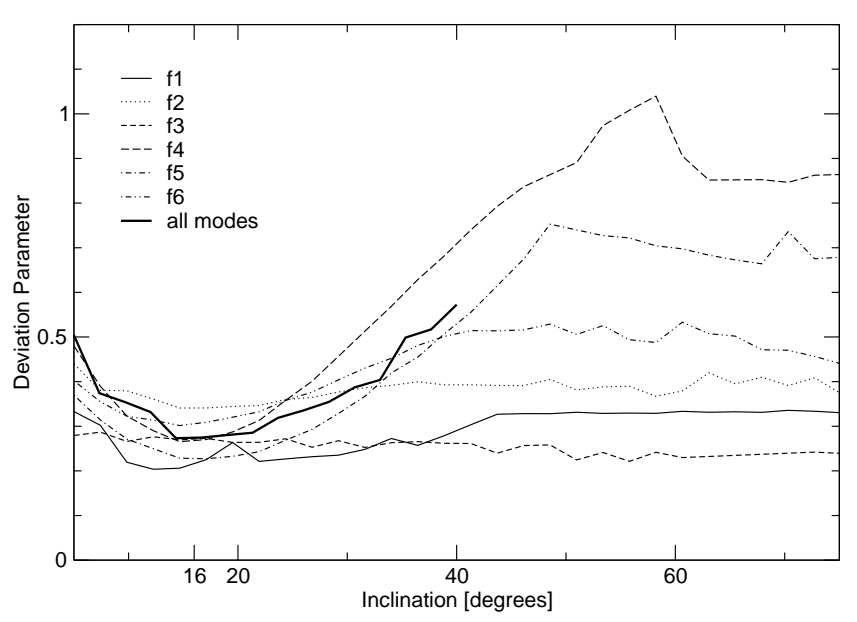

Figure 4: Dependence of the deviation parameters (arbitrary units) derived from the profile fits on the inclination of the model. For every inclination only the lowest deviation parameter calculated for the specific mode is shown. An important feature is that for the two axisymmetric modes we derive significantly wrong inclinations, which mainly is the consequence of a low dependence of the deviation parameter on the aspect angle. In contrast, the non-axisymmetric modes all show a minimum of the deviation parameter at about 16 degrees. An improvement is made by fitting all six modes simultaneously with common inclination. To save computation time, we limited the inclination value for the multi-mode fit to the range $\left[5^{\circ}, 40^{\circ}\right]$.

We determined the equivalent width, $\sigma$ and $v \sin i$ (see Table 2) from the lines by assuming that the residual line profile (zeropoint of the six-frequency least-squares fit) represents that of a non-pulsating star. Due to the fact that the line is slightly broadened by the pulsation, usually $v \sin i$ is overestimated, but can still yield reliable values, if the maximum pulsational velocity is only a fraction of the rotational broadening. Thus we stress that analyzing the 
deconstructed mono-mode line profiles only yields reliable results if the radial velocity amplitude is below $0.2 v \sin i$.

$$
\begin{gathered}
v \sin i=22.7 \pm 0.3 \mathrm{kms}^{-1} \\
\sigma=7.76 \pm 0.3 \mathrm{kms}^{-1} \\
\mathrm{EQW}=6.45 \pm 0.1 \mathrm{kms}^{-1}
\end{gathered}
$$

\begin{tabular}{c|c|c|c|c|c}
\hline name & $\ell$ & $m$ & $\frac{d R}{R}$ & Inclination & $\Gamma$ \\
\hline$f_{1}$ & $\mathbf{1}$ & $\mathbf{0}$ & 0.00158 & 22 & 0.22 \\
& 0 & 0 & 0.00210 & - & 0.32 \\
& 2 & 0 & 0.00158 & 15 & 2.30 \\
& 1 & -1 & 0.00158 & 80 & 7.22 \\
\hline$f_{2}$ & $\mathbf{1}$ & $\mathbf{0}$ & 0.00098 & 58 & 0.20 \\
& 0 & 0 & 0.00072 & - & 0.24 \\
& 2 & 0 & 0.00055 & 10 & 0.45 \\
& 1 & 1 & 0.00045 & 72 & 1.15 \\
\hline$f_{3}$ & $\mathbf{2}$ & $\mathbf{1}$ & 0.00039 & 15 & 0.27 \\
& 1 & 1 & 0.00052 & 15 & 0.32 \\
& 3 & 1 & 0.00046 & 17 & 0.43 \\
& 3 & 2 & 0.00082 & 70 & 0.85 \\
\hline$f_{4}$ & $\mathbf{3}$ & $\mathbf{- 1}$ & 0.00046 & 17 & 0.23 \\
& 2 & -1 & 0.00036 & 15 & 0.35 \\
& 1 & -1 & 0.00074 & 5 & 0.48 \\
& 3 & -2 & 0.00061 & 68 & 0.66 \\
\hline$f_{5}$ & 2 & 1 & 0.00051 & 15 & 0.30 \\
& $\mathbf{1}$ & $\mathbf{1}$ & 0.00078 & 15 & 0.30 \\
& 3 & 2 & 0.00135 & 78 & 0.42 \\
& 2 & 2 & 0.00046 & 51 & 0.50 \\
\hline$f_{6}$ & $\mathbf{3}$ & $\mathbf{- 2}$ & 0.00038 & 15 & 0.34 \\
& 3 & -1 & 0.00076 & 58 & 0.37 \\
& 2 & -2 & 0.00088 & 7 & 0.38 \\
& 3 & -3 & 0.00018 & 75 & 0.38 \\
\hline & & & &
\end{tabular}

Table 2: Best solutions of the mono-mode fits, derived from the direct fit to the mono-mode line profiles using genetic optimization. All modes were fitted with the same values of $v \sin i, \sigma$ and equivalent width. The deviation parameter $\Gamma$ is given in arbitrary units. For radial modes we did not give an inclination angle due to their spherically symmetric properties.

For every mode fitting procedure with the genetic code we had four free parameters (limits given in brackets): $\ell[0,4], m[-4,4], \frac{d R}{R}[0.00001,0.002]$ and inclination $i[5,80]$. The result of this mode identification is documented in 


\begin{tabular}{c|ccccc}
\hline$\left(\ell_{1}, m_{1}\right)$ & $(1,0)$ & $(1,0)$ & $(1,0)$ & $(1,0)$ & $(1,0)$ \\
$\left(\ell_{2}, m_{2}\right)$ & $(0,0)$ & $(0,0)$ & $(0,0)$ & $(0,0)$ & $(1,0)$ \\
$\left(\ell_{3}, m_{3}\right)$ & $(2,1)$ & $(2,1)$ & $(2,1)$ & $(2,1)$ & $(2,1)$ \\
$\left(\ell_{4}, m_{4}\right)$ & $(3,-1)$ & $(3,-1)$ & $(3,-1)$ & $(3,-1)$ & $(3,-1)$ \\
$\left(\ell_{5}, m_{5}\right)$ & $(1,1)$ & $(1,1)$ & $(2,1)$ & $(2,1)$ & $(2,1)$ \\
$\left(\ell_{6}, m_{6}\right)$ & $(3,-2)$ & $(2,-2)$ & $(2,-2)$ & $(3,-2)$ & $(3,-2)$ \\
$\frac{d R}{R}$ & 0.00152 & 0.00152 & 0.00152 & 0.00152 & 0.00158 \\
$\frac{d R}{R} 2$ & 0.00071 & 0.00071 & 0.00071 & 0.00065 & 0.00052 \\
$\frac{d R}{R}$ & 0.0004 & 0.0004 & 0.0004 & 0.0004 & 0.00034 \\
$\frac{d R}{R} 4$ & 0.00046 & 0.00046 & 0.00046 & 0.00046 & 0.00046 \\
$\frac{d R}{R} 5$ & 0.00076 & 0.00076 & 0.00058 & 0.00058 & 0.00052 \\
$\frac{d R}{R} 6$ & 0.0004 & 0.00052 & 0.00052 & 0.00034 & 0.00034 \\
$i$ & 14 & 17 & 14 & 17 & 19 \\
$\Gamma$ & 1.64 & 1.65 & 1.67 & 1.68 & 1.72 \\
\hline
\end{tabular}

Table 3: The five best solutions of the mode identification through the sum of deviation parameters $\Gamma$ for the test-case with the multi-mode direct line profile fitting for 6 modes. $\frac{d R}{R}{ }_{i}$ is the relative radius variation and $i$ is the inclination angle. Note that the derived amplitudes very well match the "observed" values.

Table 2, where the mode parameters of the four best-fitting models are listed together with the corresponding deviation parameter. For all six modes, the correct azimuthal order $m$ appears at the first place, despite the fact that the modes of low amplitude did not reach a significant SNR in the Fourier analyses. No definite statement could be made about the degree $\ell$ of most modes since for different $\ell$ values a scaling of the pulsation amplitude can yield the same line profile variations and due to the low inclination angle, modes of identical $m$ but different degree produce similar line profile variations.

The range of the possible inclination angle, determined from the mono-mode profiles is very large, and therefore we tried to better constrain this value and the harmonic degrees by simultaneously minimizing the sum of deviation parameters of all six modes with a common value of the aspect angle in the range of $\left[5^{\circ}, 40^{\circ}\right]$. To limit the size of the parameter space, we fixed the azimuthal numbers to the most probable values, derived from the fits of the single profiles. The results are listed in Table 3. As for these calculations the parameter space is very extended, more than 30000 models had to be calculated for the deviation parameter to converge. Optimizing only the four non-axisymmetric modes might be a better approach for determining the inclination, due to the larger dependence of their deviation parameter on the aspect angle (see Fig. 3). Nevertheless, the first placed model correctly identifies $\ell$ and $m$ for all modes 
except $f_{2}$, and the results of this multi-mode fit yields towards an inclination value of $15^{\circ} \pm 4^{\circ}$, which is close to the true value of $16^{\circ}$. On the other hand, it is still not possible to discriminate between different $\ell$ values.

\section{Mode Identification with the Moment Method}

The idea behind the Moment Method is that each line profile can be sufficiently described by its first three moments, which describe respectively the centroid velocity of the line, the line width and the line skewness. The wavenumbers $(\ell, m)$ and the other velocity parameters are then determined by minimizing the difference between the theoretically computed moment variations and the observed ones. The Moment Method was first introduced by Balona (1986ab, 1987) and was further developed by Aerts et al. (1992) and Aerts (1996).

Recently, motivated by the efficiency of current computers, Briquet \& Aerts (2003) developed a new version of the Moment Method, optimized for mode identification in multi-periodic stars. The new version requires less computation time than the previous one, and allows users to consider all information contained in the first three moments of a multi-periodic star and to identify multiple modes simultaneously, leading to only one derived value for the continuous parameters such as the inclination. Moreover, the new method considers the moments calculated at each time of observation, instead of considering the amplitudes derived from the whole data set. We applied this new version of the Moment Method to our data set.

\begin{tabular}{ccc}
\hline & Amplitude $\left(\mathrm{km} \mathrm{s}^{-1}\right)$ & Phase (degrees) \\
\hline$f_{1}$ & $2.103 \pm 0.026$ & $29.2 \pm 0.7$ \\
$f_{2}$ & $0.707 \pm 0.026$ & $121.6 \pm 2.1$ \\
$f_{3}$ & $0.237 \pm 0.026$ & $46.2 \pm 6.2$ \\
$f_{4}$ & $0.125 \pm 0.026$ & $305.8 \pm 11.7$ \\
$f_{5}$ & $0.162 \pm 0.026$ & $73.8 \pm 9.1$ \\
$f_{6}$ & $0.029 \pm 0.026$ & $159.4 \pm 50.6$ \\
\hline
\end{tabular}

Table 4: Amplitudes and phases of the least-squares sine fits to the first moment (radial velocity) for the six frequencies. The errors were calculated following Breger (1999).

Because $f_{6}$ has a low amplitude in the moments compared to the other frequencies, we decided to perform a mode identification for the five modes with highest amplitudes. The amplitudes for the first moment (radial velocity) 
are listed in Table 4. To limit the parameter space, ranges for the projected rotational velocity $v \sin i$ and the intrinsic line profile width $\sigma$ were determined by a line profile fitting to the "constant" profile derived as the mean of all profiles, as described in the previous section. We varied $v \sin i$ from 22 to 24 $\mathrm{km} \mathrm{s}^{-1}$ with a step $0.1 \mathrm{~km} \mathrm{~s}^{-1}$ and $\sigma$ from 5 to $10 \mathrm{~km} \mathrm{~s}^{-1}$ with a step 0.1 $\mathrm{km} \mathrm{s}^{-1}$. The inclination angle $i$ was varied from $0^{\circ}$ to $90^{\circ}$ with a step $1^{\circ}$. The Moment Method is implemented for a linear limb darkening and we took a linear coefficient $u$ of 0.55 in order to correspond as much as possible to the second order limb darkening. We tested all combinations of modes for $0 \leq \ell \leq 4$ and $|m| \leq \ell$. In order to reduce considerably the number of tested combinations of modes, we used the constraint on the sign of $m$ given by the Pixel-by-Pixel Method (see Fig. 2). The outcome of this multi-periodic mode identification is displayed in Table 5 in which the right combinations of modes is listed as solution 8. All best solutions with an almost equal value for the discriminant pinpoint the right values for $m$, which are $1,-1$ and 1 respectively for $m_{3}, m_{4}$ and $m_{5}$. The inclination is determined as $i=14^{\circ} \pm 2^{\circ}, v \sin i$ is overestimated while $\sigma$ is underestimated. The equivalent best solutions correspond to the different combinations with $\ell_{1}=1, \ell_{2}$ and $\ell_{3}$ being 1 or $2, \ell_{4}$ being 1 or 2 or 3 and $\ell_{5}$ being 1 or 2 . Note that for such a low value of the inclination, modes with same $m$ but different $\ell$ have a very similar visible configuration, which might explain why the method does not succeed in discriminating the degree $\ell$ unambiguously. For the correct 5-mode solution, appearing on the eighth place in the table, the relative radius variations of the modes are determined quite well.

We also performed a simultaneous mode identification for the six modes in a similar way but varying $i$ with a step $5^{\circ}$ for limiting computation time. The outcome is shown in Table 6 . The sixth mode is not at all well identified, which may be explained by the fact that this mode does not appear clearly in the moments, where its amplitude is not significant. For the other modes, conclusions are similar to the ones taking into account five modes only.

Note that the version of the Moment Method that we used does not take into account the effect of the Coriolis force. However, given the small ratio of rotation and pulsation frequencies, the effects of the Coriolis force are small and will not influence much the results. Moreover, the second order limb darkening and temperature variations are not yet implemented. However, as these latter effects are rather small on the normalized moments, the results are quite good.

\section{A faster combination of both mode identification techniques?}

In the Pixel-by-Pixel Method we determined the amplitude and phase variations across the line profile for each of the detected frequencies (see Fig. 2). By adding 


\begin{tabular}{c|cccccccccc}
$\left(\ell_{5}, m_{5}\right)$ & $(1,1)$ & $(1,1)$ & $(2,1)$ & $(2,1)$ & $(2,1)$ & $(2,1)$ & $(2,1)$ & $(1,1)$ & $(1,1)$ & $(1,1)$ \\
$\frac{d R}{R}$ & 0.00147 & 0.00147 & 0.00149 & 0.00149 & 0.00147 & 0.00148 & 0.00148 & 0.00149 & 0.00148 & 0.00148 \\
$\frac{d R}{R} 2$ & 0.00049 & 0.00049 & 0.00064 & 0.00050 & 0.00049 & 0.00049 & 0.00049 & 0.00050 & 0.00049 & 0.00049 \\
$\frac{d R}{R} 3$ & 0.00066 & 0.00071 & 0.00044 & 0.00041 & 0.00066 & 0.00061 & 0.00047 & 0.00044 & 0.00047 & 0.00061 \\
$\frac{d R}{R} 4$ & 0.00026 & 0.00036 & 0.00034 & 0.00033 & 0.00026 & 0.00036 & 0.00024 & 0.00034 & 0.00024 & 0.00036 \\
$\frac{d R}{R}{ }_{5}$ & 0.00091 & 0.00099 & 0.00053 & 0.00050 & 0.00060 & 0.00056 & 0.00056 & 0.00079 & 0.00085 & 0.00085 \\
$i$ & 13 & 12 & 15 & 16 & 13 & 14 & 14 & 15 & 14 & 14 \\
$v \sin i$ & 23.9 & 23.9 & 22.7 & 23.9 & 23.8 & 23.9 & 23.9 & 23.9 & 23.9 & 23.9 \\
$\sigma$ & 6.7 & 6.7 & 7.6 & 6.7 & 6.8 & 6.7 & 6.7 & 6.7 & 6.7 & 6.7 \\
$\Sigma$ & 7.721 & 7.722 & 7.722 & 7.723 & 7.723 & 7.723 & 7.724 & 7.724 & 7.724 & 7.724 \\
\hline
\end{tabular}

Table 5: The ten best solutions of the mode identification through the discriminant $\Sigma$ for the test-case with the multi-periodic Moment Method (Briquet \& Aerts 2003) for 5 modes. $\frac{d R}{R}{ }_{i}$ is the relative radius variation; $i$ is the inclination angle; $v$ sin $i$ is the projected rotational velocity, expressed in $\mathrm{km} \mathrm{s}^{-1}$ and $\sigma$ is the intrinsic line-profile width, also expressed in $\mathrm{km} \mathrm{s}^{-1}$. The correct combination of modes appears on place eight. Note that the solutions up to the twelfth place have approximately the same discriminant value (7.724) as the right solution. 


\begin{tabular}{|c|c|c|c|c|c|c|c|c|c|c|}
\hline$\left(\ell_{6}, m_{6}\right)$ & $(4,-1)$ & $(4,-1)$ & $(4,-1)$ & $(4,-1)$ & $(4,-1)$ & $(3,-1)$ & $(3,-1)$ & $(3,-1)$ & $(4,-1)$ & $(4,-1)$ \\
\hline$\frac{d R}{R}{ }_{1}$ & 0.00149 & 0.00149 & 0.00149 & 0.00149 & 0.00149 & 0.00149 & 0.00149 & 0.00149 & 0.00149 & 0.00153 \\
\hline$\frac{d R}{R}{ }_{2}$ & 0.00050 & 0.00050 & 0.00064 & 0.00064 & 0.00064 & 0.00050 & 0.00050 & 0.00050 & 0.00064 & 0.00051 \\
\hline$d$ & 0.00044 & 0.00044 & 0.00044 & 0.00044 & 0.00057 & 0.00044 & 0.00044 & 0.00044 & 0.00057 & 0.00054 \\
\hline$\frac{d R}{R} 4$ & 0.00034 & 0.00034 & 0.00034 & 0.00034 & 0.00034 & 0.00034 & 0.00022 & 0.00034 & 0.00034 & 0.00028 \\
\hline$\frac{d R}{R}{ }_{5}$ & 0.00079 & 0.00053 & 0.00053 & 0.00079 & 0.00053 & 0.00053 & 0.00053 & 0.00079 & 0.00079 & 0.00056 \\
\hline$\frac{d R}{R}_{6}$ & 0.00035 & 0.00035 & 0.00035 & 0.00035 & 0.00035 & 0.00009 & 0.00009 & 0.00009 & 0.00035 & 0.00030 \\
\hline$i$ & 15 & 15 & 15 & 15 & 15 & 15 & 15 & 15 & 15 & 20 \\
\hline$v \sin i$ & 23.9 & 23.9 & 22.9 & 23.1 & 22.9 & 23.9 & 23.9 & 23.9 & 23.1 & 23.8 \\
\hline$\sigma$ & 6.7 & 6.7 & 7.4 & 7.3 & 7.4 & 6.7 & 6.7 & 6.7 & 7.3 & 6.7 \\
\hline$\Sigma$ & 7.724 & 7.725 & 7.725 & 7.725 & 7.726 & 7.726 & 7.727 & 7.727 & 7.727 & 7.727 \\
\hline
\end{tabular}

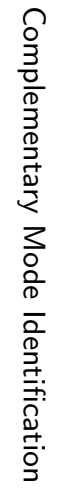

Table 6: The ten best solutions of the mode identification through the discriminant $\Sigma$ for the test-case with the multi-periodic Moment Method (Briquet \& Aerts 2003) for 6 modes. $\frac{d R}{R}{ }_{i}$ is the relative radius variation; $i$ is the inclination angle; $v$ sin $i$ is the projected rotational velocity, expressed in $\mathrm{km} \mathrm{s}^{-1}$ and $\sigma$ is the intrinsic line-profile width, also expressed in $\mathrm{km} \mathrm{s}^{-1}$. 
the amplitude and phase variations over the profile to the mean profile (the socalled "constant" profile without any variation), 10 mono-mode profiles were constructed, evenly distributed over one pulsation cycle, for every single mode. We stress that this reconstruction does not work for modes with amplitudes large compared to $v \sin i$, while there the mean or "constant" profile will be distorted.

As we have then, for each mode, line profile variations over one pulsation cycle, we can calculate moments and apply the Moment Method (Briquet \& Aerts 2003) for a mono-periodic pulsation to each of the modes separately. As the frequencies of the different modes are accurately known, only a limited amount of "reconstructed profiles" per mode is needed to perform an accurate fit of the moments, yielding calculation times of only a few seconds.

Each application yields the most probable solution for the considered mode, as well as the best-fitting values for the continuous parameters $i, v \sin i$, and $\sigma$. Subsequently, the results for the separate modes are combined by searching for the smallest summed discriminant for all considered modes together with common $i, v \sin i, \sigma$. This combining of the output of the different monoperiodic programs takes of the order of minutes, depending on the scanned grid.

The results for each of the detected modes separately as well as the combined results for common continuous parameters are given in Tables 7,8 and 9. Note that we combined the results for 5 modes (Table 8) and for 6 modes (Table 9). The inclusion of the sixth mode is now justified by the fact that this mode has a significant amplitude in the pixel-to-pixel variations, whereas its amplitude in the first moment was insignificant (see previous section). The limited calculation time - less than one hour to come to the combined results for all modes - represents a large advantage. We did not impose any constraints on the sign or value of $m$ for all modes with $\ell \leq 4$. From Tables 7, 8 and 9 can be seen that the correct sign, and except for $f_{6}$ also the correct value, of $m$ was easily determined by the codes. The results shown in Tables 7,8 and 9 were calculated for $v \sin i$ from 21 to $25 \mathrm{~km} \mathrm{~s}^{-1}$ with a step of $0.1 \mathrm{~km} \mathrm{~s}^{-1}$, and $\sigma$ from 5 to $10 \mathrm{~km} \mathrm{~s}^{-1}$ with a step of $0.1 \mathrm{~km} \mathrm{~s}^{-1}$, while $i$ was varied from $0^{\circ}$ to $90^{\circ}$ with a step $1^{\circ}$.

The mono-mode solutions (Table 7 ), obtained in very little time, are quite encouraging: the correct mode solution always appears; if not on the first place, then with a discriminant only differing slightly from the smallest discriminant. These results can be compared with the ones presented in Table 2, where a line profile fitting was applied to the same profiles in order to fix the best fitting modes. For the axisymmetric modes related to $f_{1}$ and $f_{2}$, the inclination is not well determined, as was also the case with the Pixel-by-Pixel Method. For the other modes, the best fitting inclination is very close to the real value of $16^{\circ}$. 


\begin{tabular}{c|c|c|c|c|c|c|c}
\hline name & $\ell$ & $m$ & $\frac{d R}{R}$ & $i$ & $v \sin i$ & $\sigma$ & $\Sigma$ \\
\hline$f_{1}$ & $\mathbf{1}$ & $\mathbf{0}$ & 0.00144 & 5 & 22.5 & 7.8 & 2.02 \\
& 0 & 0 & 0.00204 & - & 24.6 & 6.0 & 2.95 \\
& 2 & 0 & 0.00174 & 4 & 21.0 & 8.8 & 4.41 \\
& 1 & -1 & 0.00203 & 90 & 21.0 & 8.4 & 4.55 \\
\hline$f_{2}$ & $\mathbf{1}$ & $\mathbf{0}$ & 0.00057 & 33 & 22.9 & 7.7 & 1.31 \\
& 0 & 0 & 0.00068 & - & 24.9 & 6.1 & 2.16 \\
& 1 & 1 & 0.00067 & 90 & 22.0 & 8.2 & 2.55 \\
& 1 & -1 & 0.00067 & 90 & 22.4 & 7.9 & 2.57 \\
\hline$f_{3}$ & 1 & 1 & 0.00061 & 14 & 22.3 & 8.1 & 1.31 \\
& $\mathbf{2}$ & $\mathbf{1}$ & 0.00044 & 15 & 21.1 & 8.8 & 1.43 \\
& 3 & 1 & 0.00056 & 19 & 21.1 & 8.8 & 2.41 \\
& 1 & 0 & 0.00300 & 88 & 22.0 & 7.7 & 2.77 \\
\hline$f_{4}$ & $\mathbf{3}$ & $\mathbf{- 1}$ & 0.00034 & 16 & 23.2 & 7.5 & 1.36 \\
& 2 & -1 & 0.00027 & 13 & 24.5 & 6.5 & 1.92 \\
& 1 & -1 & 0.00038 & 12 & 24.5 & 6.5 & 2.14 \\
& 2 & -2 & 0.00012 & 80 & 21.0 & 8.9 & 2.17 \\
\hline$f_{5}$ & $\mathbf{1}$ & $\mathbf{1}$ & 0.00105 & 11 & 22.3 & 8.1 & 1.29 \\
& 2 & 1 & 0.00063 & 12 & 21.1 & 8.8 & 1.35 \\
& 3 & 1 & 0.00061 & 17 & 21.1 & 8.8 & 2.00 \\
& 0 & 0 & 0.00021 & - & 23.1 & 7.6 & 2.38 \\
\hline$f_{6}$ & 3 & -3 & 0.00050 & 31 & 23.2 & 7.5 & 3.21 \\
& 2 & -2 & 0.00052 & 14 & 23.2 & 7.5 & 3.21 \\
& $\mathbf{3}$ & $\mathbf{- 2}$ & 0.00038 & 16 & 21.6 & 8.5 & 3.21 \\
& 4 & -3 & 0.00041 & 54 & 22.1 & 8.2 & 3.22 \\
\hline \multirow{6}{*}{} & & & & & & \\
& & & &
\end{tabular}

Table 7: Mode parameters derived for the mono-mode profiles from the mono-periodic Moment Method. The right solutions for $\ell$ and $m$ are displayed in bold face. $\frac{d R}{R}{ }_{i}$ is the relative radius variation; $i$ is the inclination angle; $v \sin i$ is the projected rotational velocity, expressed in $\mathrm{km} \mathrm{s}^{-1}$ and $\sigma$ is the intrinsic line-profile width, also expressed in $\mathrm{km} \mathrm{s}^{-1}$. For radial modes we did not give an inclination angle due to their spherically symmetric properties.

When combining the results of the mono-mode moment fits for common $i, v \sin i$ and $\sigma$, we observe that the correct values of $m$ are determined unambiguously. Just like in the mono-mode solutions, there is some ambiguity in 


\begin{tabular}{c|cccccccccc}
$\left(\ell_{5}, m_{5}\right)$ & $(1,1)$ & $(2,1)$ & $(1,1)$ & $(2,1)$ & $(\mathbf{1 , 1})$ & $(1,1)$ & $(1,1)$ & $(0,0)$ & $(1,1)$ & $(2,1)$ \\
$\frac{d R}{R}{ }_{1}$ & 0.00148 & 0.00148 & 0.00146 & 0.00149 & 0.00151 & 0.00145 & 0.00145 & 0.00152 & 0.00144 & 0.00147 \\
$\frac{d R}{R} 2^{2}$ & 0.00049 & 0.00049 & 0.00048 & 0.00050 & 0.00050 & 0.00048 & 0.00048 & 0.00050 & 0.00048 & 0.00049 \\
$\frac{d R}{R}{ }_{3}$ & 0.00057 & 0.00057 & 0.00078 & 0.00041 & 0.00037 & 0.00107 & 0.00107 & 0.00046 & 0.00011 & 0.00071 \\
$\frac{d R}{R}{ }_{4}$ & 0.00036 & 0.00036 & 0.00031 & 0.00034 & 0.00031 & 0.00057 & 0.00007 & 0.00030 & 0.00007 & 0.00029 \\
$\frac{d R}{R}{ }_{5}$ & 0.00077 & 0.00051 & 0.00105 & 0.00048 & 0.00065 & 0.00144 & 0.00144 & 0.00021 & 0.00164 & 0.00063 \\
$i$ & 15 & 15 & 11 & 16 & 18 & 8 & 8 & 19 & 7 & 12 \\
$v \sin i$ & 23.2 & 22.6 & 24.0 & 21.8 & 24.8 & 23.3 & 23.5 & 24.7 & 23.3 & 24.8 \\
$\sigma$ & 7.5 & 7.9 & 6.9 & 8.4 & 6.3 & 7.4 & 7.3 & 6.3 & 7.4 & 6.3 \\
$\Sigma$ & 8.105 & 8.528 & 9.649 & 9.664 & 10.642 & 10.673 & 10.721 & 10.753 & 10.944 & 10.973 \\
\hline
\end{tabular}

Table 8: The ten best solutions of the mode identification through the discriminant $\Sigma$ for our test-case by combining the monomode solutions for five modes. $\frac{d R}{R}{ }_{i}$ is the relative radius variation; $i$ is the inclination angle; $v \sin i$ is the projected rotational velocity, expressed in $\mathrm{km} \mathrm{s}^{-1}$ and $\sigma$ is the intrinsic line-profile width, also expressed in $\mathrm{km} \mathrm{s}^{-1}$. The right combination of modes appears on the fifth place. 


\begin{tabular}{c|cccccccccccc}
$\left(\ell_{5}, m_{5}\right)$ & $(1,1)$ & $(1,1)$ & $(2,1)$ & $(1,1)$ & $(2,1)$ & $(2,1)$ & $(1,1)$ & $(1,1)$ & $(1,1)$ & $(2,1)$ & $(1,1)$ & $(\mathbf{1}, \mathbf{1})$ \\
$\left(\ell_{6}, m_{6}\right)$ & $(2,-2)$ & $(3,-2)$ & $(2,-2)$ & $(4,0)$ & $(3,-2)$ & $(3,-2)$ & $(4,0)$ & $(4,-2)$ & $(3,-3)$ & $(2,-2)$ & $(2,-2)$ & $(\mathbf{3},-\mathbf{2})$ \\
$\frac{d R}{R}{ }^{2}$ & 0.00148 & 0.00150 & 0.00148 & 0.00147 & 0.00150 & 0.00149 & 0.00146 & 0.00166 & 0.00167 & 0.00148 & 0.00147 & 0.00151 \\
$\frac{d R}{R}{ }_{2}$ & 0.00049 & 0.00050 & 0.00049 & 0.00049 & 0.00050 & 0.00049 & 0.00048 & 0.00055 & 0.00056 & 0.00049 & 0.00049 & 0.00050 \\
$\frac{d R}{R}{ }_{3}$ & 0.00057 & 0.00051 & 0.00057 & 0.00066 & 0.00051 & 0.00041 & 0.00078 & 0.00030 & 0.00029 & 0.00044 & 0.00066 & 0.00037 \\
$\frac{d R}{R} 4$ & 0.00036 & 0.00032 & 0.00036 & 0.00040 & 0.00032 & 0.00034 & 0.00031 & 0.00025 & 0.00025 & 0.00036 & 0.00027 & 0.00031 \\
$\frac{d R}{R} 5$ & 0.00077 & 0.00068 & 0.00051 & 0.00089 & 0.00046 & 0.00048 & 0.00105 & 0.00040 & 0.00039 & 0.00051 & 0.00089 & 0.00065 \\
$\frac{d R}{R}{ }^{R}$ & 0.00046 & 0.00034 & 0.00046 & 0.00025 & 0.00034 & 0.00038 & 0.00023 & 0.00044 & 0.00050 & 0.00046 & 0.00061 & 0.00031 \\
$i$ & 15 & 17 & 15 & 13 & 17 & 16 & 11 & 30 & 31 & 15 & 13 & 18 \\
$v \sin i$ & 23.5 & 23.2 & 22.6 & 23.6 & 22.6 & 21.8 & 24.0 & 23.3 & 23.3 & 21.1 & 24.8 & 24.8 \\
$\sigma$ & 7.3 & 7.5 & 7.9 & 7.2 & 7.9 & 8.4 & 6.9 & 7.4 & 7.4 & 8.8 & 6.2 & 6.3 \\
$\Sigma$ & 11.367 & 11.628 & 11.807 & 11.953 & 12.108 & 12.896 & 13.075 & 13.137 & 13.170 & 13.257 & 13.903 & 13.925
\end{tabular}

Table 9: The twelve best solutions of the mode identification through the discriminant $\Sigma$ for our test-case by combining the mono-mode solutions for six modes. $\frac{d R}{R}{ }_{i}$ is the relative radius variation; $i$ is the inclination angle; $v \sin i$ is the projected rotational velocity, expressed in $\mathrm{km} \mathrm{s}^{-1}$ and $\sigma$ is the intrinsic line-profile width, also expressed in $\mathrm{km} \mathrm{s}^{-1}$. The right combination of modes appears on the twelfth place. 
the determination of $\ell_{3}$, so that the correct mode combination appears 'only' on the fifth place in the 5-mode solution (Table 8) and on the twelfth place in the 6 -mode solution (Table 9 ). The inclusion of $f_{6}$ in the calculations make the determination of the correct $\ell$ values more ambiguous, though we still find values close to the real ones, also for the continuous parameters, like the modes' amplitudes. For the tabulated results the value of $v \sin i$ is overestimated while $\sigma$ is underestimated. In the combined results, $i$ is only slightly overestimated.

Though the time-saving combination of Pixel-by -Pixel method and Moment Method yields rather encouraging results for the considered test-case, more tests are definitely needed to probe the consistency of both Moment Method applications, and determine the constraints of applicability of this faster combined method. In any case, an application of the combined method, should always be accompanied by the two other methods, of which the reliability has been proven.

\section{Conclusions}

Mode identification still remains a stubborn problem and therefore any new ideas about it should be tested and worked out. In this paper we applied two different types of spectroscopic identification methods, the Pixel-by-Pixel Method and the Moment Method, on theoretically generated "realistic" line profiles of a Delta Scuti-like star with 6 pulsation modes. The results open perspectives for the identification of multiple pulsation modes in Delta Scuti stars.

As both methods yield some tests to limit the parameter space, it is advisable to exchange information between them, e.g. by using the additional constraint on $m$ given by the phase diagrams in the Pixel-by-Pixel Method, or the constraint on the velocity amplitude given by the moment variations. Whereas the sign and value of $m$ could be fixed by both methods, the degree $\ell$ is not determined unambiguously. The inclination angle can be better determined if multiple modes are fitted simultaneously. We also applied a combination of the Pixel-by-Pixel Method and the (mono-mode) Moment Method, requiring much less computation time, and yielding comparable results. The applicability of this combined technique strongly depends on the ratio between the rotational velocity and the pulsational velocities of the star. We emphasize that the outcome of this test is only meaningful for stars having pulsational velocities below $0.2 v \sin i$.

This is the first part of a number of projected articles, in which we will test 
and extend these spectroscopic identification techniques.

Acknowledgments. M. Briquet is Postdoctoral Fellow of the Fund for Scientific Research, Flanders. This work is supported by the Austrian Fonds zur Förderung der wissenschaftliche Forschung (FWF) within the project Stellar Seismology (P14546-Phy). We thank Conny Aerts for proofreading this article.

\section{References}

Aerts, C., De Pauw M., Waelkens C. 1992, A\&A, 266, 294

Aerts, C. 1996, A\&A, 314, 115

Aerts, C., Eyer, L. 2000, in "Delta Scuti and Related Stars", eds. Breger,M., Montgomery, M.H., ASP Conf. Ser. 210, 113

Balona, L.A. 1986a, MNRAS, 219, 111

Balona, L.A. 1986b, MNRAS, 220, 647

Balona, L.A. 1987, MNRAS, 224, 41

Balona, L.A. 2000, in "Delta Scuti and Related Stars", eds. Breger, M., Montgomery, M.H., ASP Conf. Ser. 210, 170

Breger, M. 1993, in Butler, C.J., Elliott, I., eds., Stellar Photometry - Current Techniques and Future Developments, Cambridge Univ. Press, 106

Breger, M. et al. 1998, A\&A 331, 271

Breger, M. et al. 1999, A\&A 349, 225

Briquet, M., Aerts, C. 2003, A\&A 398, 687

Mantegazza, L. 2000, in "Delta Scuti and Related Stars", eds. Breger, M., Montgomery, M.H., ASP Conf. Ser. 210, 138

Schrijvers, C., Telting, J.H., Aerts, C., Ruymaekers, E., Henrichs, H.F. 1997, A\&AS 121, 343

Schrijvers, C., Telting, J.H. 1999, A\&A 342, 453

Zima, W. et al. 2003, in "Asteroseismology Across the HR Diagram",

A\&SS 284, 489 